\title{
Toll-Like Receptor-2 Ligand Peptidoglycan Upregulates Expression and Ubiquitin Ligase Activity of CHIP through JNK Pathway
}

\author{
Yan Meng ${ }^{\mathrm{a}}$ Chen Chen ${ }^{\mathrm{b}}$ Lei Wang ${ }^{\mathrm{a}}$ Xia Wang ${ }^{\mathrm{a}}$ Cui Tian ${ }^{\mathrm{a}} \quad$ Jie Dub Hui-Hua Lia \\ aDepartment of Pathology, Physiology and Pathophysiology, AnZhen Hospital the Key Laboratory \\ of Remodeling-Related Cardiovascular Diseases, School of Basic Medical Sciences, Capital Medical \\ University, Beijing, 'beijing AnZhen Hospital, Capital Medical University, Beijing Institute of Heart, \\ Lung and Blood Vessel Diseases, Beijing, China
}

\section{Key Words}

CHIP • Peptidoglycan • Inflammation • Ubquitination • JNK

\begin{abstract}
Background: Peptidoglycan (PGN) is a component of cell wall in Gram-positive bacteria that stimulates inflammatory responses through Toll-like receptor 2 (TLR2). The carboxyl terminus of constitutive heat shock cognate 70 (HSC70)-interacting protein (CHIP, also known as Stub1) is a U-box-type E3 ubiquitin ligase, which plays an important role in protein quality control and inflammation through ubquitin-mediated proteasomal degradation. However, it is unclear whether TLR2 agonist PGN regulates the expression and activation of CHIP. Methods/Results: In this study, we showed that PGN significantly up-regulated the expression of CHIP in both mRNA and protein levels in RAW264.7 cells in a time-dependant manner, and the expression of CHIP induced by PGN was abolished in TLR2 knockout macrophages. No significant change in CHIP was observed after lipopolysaccharide (LPS, TLR4 agonist) and cytosinephosphorous-guanine oligonucleotide (CPG ODN, TLR9 agonist) treatment. Moreover, PGN markedly induced the expression and activity of CHIP in macrophages, whereas this effect was attenuated by SP600125, a selective inhibitor of JNK. Conclusion: Our study for the first time demonstrates that TLR2 activation enhances the expression and activity of CHIP through JNK signaling pathway.
\end{abstract}




\section{Introduction}

Sepsis is caused by the immune system's response to a serious infection, most commonly bacteria. Despite substantial prevention efforts to improve patient outcome, Gram-positive bacteria are still the predominant organisms in sepsis case since 19th century [1]. Toll-like receptors (TLRs) are pattern recognition receptors (PRRs) of innate immune system that recognize conserved microbial structures called PAMPs (pathogen-associated molecular patterns), including lipopolysaccharide (LPS), peptidoglycan (PGN), cytosine-phosphorousguanine oligonucleotide (CpG ODN). LPS is a major component of the outer membrane of Gram negative bacteria, while PGN (also known as murein) serves a structural component in cell wall of Gram-positive bacteria. CpG ODNs are synthetic oligonucleotides containing CpG motifs [2]. Increasing studies have demonstrated that PAMPs (such as LPS, PGN and CpG ODNs) activates host cells through the pattern recognition receptors Toll-like receptors (such as TLR4, TLR2 and TLR9), which play important roles in both innate and adaptive immunity $[3,4]$. The MAPKs are serine/threonine signaling kinases that mediate the phosphorylation and activation of transcription factors. There are three main members of MAPK family in mammalian cells stress-activated protein kinase c-Jun NH2-terminal kinase (JNK), stressactivated protein kinase 2 (SAPK2 or p38) and the extracellular signal-regulated protein kinases (ERK1/2, p44/p42) [5]. MAPKs are activated by not only cellular stresses such as hypoxic and oxidative stress, but also proinflammatory cytokines, including TNF $\alpha$, IL-6, IL-1 or LPS [6-8]. Recently, Bhatt et al found that PGN activates MAPK pathway in mouse peritoneal macrophages, which results in the expression of inducible Nitric Oxide [9].

The ubiquitin-proteasome system (UPS) is responsible for the degradation and clearance of unneeded or damaged intracellular proteins to maintain the cell homeostasis $[10,11]$. C terminus of Hsp70-interacting protein (CHIP) is a U-box-dependent E3 ubiquitin ligase which is highly expressed in human striated muscle, aortic smooth muscle cells (SMCs), and endothelial cell [12]. Increasing evidence suggests that CHIP plays a critical role in the regulation of cell growth, apoptosis, neurodegeneration, invasion, SMC differentiation and cardiac fibrosis [13-15]. Recently, it is reported that CHIP also participates in innate immunity through TLR4 and TLR9, rather than TLR3-driven pathway. CHIP acts as an E3 ligase for Src and PKC in TLR4 and TLR9-driven signaling [16]. It has been known that the expression of CHIP can be regulated by acute injury, such as hypoxia, ischemia, ischemia/ reperfusion (I/R) injury and angiotensin II (Ang II) [13, 17, 18]. However, it is unclear whether TLR-driven pathway plays a role in the regulation of CHIP expression.

In the present study, we aimed to explore whether TLR2 signaling regulates expression and activity in the RAW264.7 macrophages. Here we demonstrated that TLR2 agonist PGN up-regulated the expression of CHIP in mRNA and protein levels in a time-dependant manner, and this effect of PGN on CHIP expression was abolished in TLR2-deficient macrophages. Moreover, PGN-induced expression and activity of CHIP were significantly attenuated by a selective inhibitor of JNK, SP600125. Thus, the present study for the first time revealed that TLR2 agonist PGN increased the expression and activity of E3 ligase CHIP by the activation of JNK signaling pathway.

\section{Materials and Methods}

\section{Antibodies and Reagents}

Dulbecco modified Eagle medium (DMEM), trypsin and fetal calf serum (FCS) were purchased from Hyclone (South Logan, UT). The anti-CHIP and anti- $\beta$-actin antibodies were purchased from Santa Cruz Biotechnology (Santa Cruz, CA). The anti-Ub antibody was purchased from Chemicon International Inc. (Temecula, CA). The anti-phospho-p38, anti-phospho-JNK, anti-phospho-ERK, anti-ERK, anti-p38, anti-JNK and anti-mouse/anti-rabbit conjugated antibodies were purchased from Cell Signaling Technology (Beverly, MA). LPS, PGN and CpG ODN were obtained from Sigma-Aldrich (St Louis, MO). SP600125 (JNK inhibitor), 
SB203580 (p38 inhibitor) and PD98059 (ERK inhibitor) were purchased from Calbiochem (La Jolla, CA). All other chemicals were from Sigma-Aldrich (St Louis, MO), unless specified otherwise.

\section{Cell culture and treatment}

Raw 264.7 cell line was purchased from ATCC and cultured in DMEM supplemented with 10\% fetal calf serum, penicillin $(100 \mathrm{U} / \mathrm{ml})$, and streptomycin $(100 \mathrm{mg} / \mathrm{ml})$ at $37^{\circ} \mathrm{C}$ under a humidified atmosphere of 95\% air and $5 \% \mathrm{CO}_{2}$. To evaluate the involvement of signaling pathways in TLR agonist-induced expression of CHIP, cells were pretreated with SB203580 (p38 MAPK inhibitor, $10 \mu \mathrm{M}$ ), PD98059 (ERK1/2 inhibitor, 10 $\mu \mathrm{M})$, SP600125 (JNK inhibitor, $10 \mu \mathrm{M}$ ) for $0.5 \mathrm{~h}$ and then treated with LPS (200 ng/ml), PGN (10 $\mu \mathrm{g} / \mathrm{ml})$ and CpG ODN $(1 \mu \mathrm{g} / \mathrm{ml})$ for the indicated time points.

Mice

Pathogen-free female TLR2\% mice and their wild-type controls were purchased from The Jackson Laboratories (Bar Harbor, ME). Animal protocols were approved by the Animal Care and Use Committee of Capital Medical University. The investigation conformed to the Guide for the Care and Use of Laboratory Animals published by the US National Institutes of Health (NIH publication no. 85-23, revised 1996).

\section{Isolation of peritoneal macrophages}

Mice (8-week-old male) were injected intraperitoneally with $3 \mathrm{ml}$ of $3 \%$ thioglycolate. After 3 days, peritoneal cells were collected by peritoneal lavage using $10 \mathrm{ml}$ ice-cold phosphate-buffered saline (PBS) , centrifuged at 1,000 rpm for $10 \mathrm{~min}$, resuspended in RPMI1640 media supplemented 10\% fetal bovine serum, and incubated on the 24-well plate for $1 \mathrm{~h}$ and then used for the experiments as described [19].

\section{Quantitative real-time PCR analysis}

Total RNA was purified from cultured cells with Trizol (Invitrogen). Specific primers used for quantitative real-time PCR (qPCR) assays were 5'-TGGAGAGTTATGATGAGGCCA-3' (sense) and 5'-GCGAAGGGCACTAGGAATATC-3' (anti-sense) for CHIP, and 5'-AGGTCGGTGTGAACGGATTTG-3' (sense) and 5'-TGTAGACCATGTAGTTGAGGTCA-3' (anti-sense) for GAPDH. qRT-PCR was performed on an iQ5 RealTime PCR Detection System (Bio-Rad Laboratories) according to the manufacturer's protocol [20].

\section{Western Blot and Immunoprecipitation analysis}

Western blot analysis was performed as previously described [17]. The cells were lysed with extraction buffer (50 mmol/L Tris-HCl pH 7.5, 0.5\% Triton X-100, 0.5\% NP40, 150 mmol/L NaCl, 2 mmol/L EDTA, 1 $\mathrm{mmol} / \mathrm{L} \mathrm{NaF}, 1 \mathrm{mmol} / \mathrm{L} \mathrm{PMSF}, 1 \mathrm{mmol} / \mathrm{L} \mathrm{Na}_{3} \mathrm{VO}_{4}$, and 10\% protease inhibitors). Fifty-sixty micrograms of extracts were separated by SDS-PAGE on a $10 \%$ gel and were transferred to a nitrocellulose membrane. The membranes were incubated with primary antibodies against CHIP (1:1000), JNK (1:500), ERK (1:1000), p38 MAPK (1:500) and $\beta$-actin (1:2000) overnight, then incubated with an HRP-conjugated secondary antibody for $1 \mathrm{~h}$. The phospho-specific proteins were normalized to total target protein and $\beta$-actin. For immunoprecipitation assays, cell lysates were precleared with protein A/G-Sepharose $(50 \%$ slurry equilibrated in phosphate-buffered saline) for $2 \mathrm{~h}$ at $4^{\circ} \mathrm{C}$ and then incubated with $5 \mu \mathrm{g}$ of anti-CHIP antibody for $4 \mathrm{~h}$ at $4^{\circ} \mathrm{C}$. Protein A/G-Sepharose was added and incubated for an additional $1 \mathrm{~h}$ at $4^{\circ} \mathrm{C}$ to capture primary immune complexes. The beads were washed and fractionated by $10 \%$ SDS-PAGE followed by immunoblotting as previously described [21, 22].

\section{Statistical analysis}

Data are presented as mean \pm SEM. Statistical analyses involved use of one-way analysis of variance analysis followed by $t$-test for multiple comparisons within treatment groups with use of SPSS v13.0 (SPSS, Chicago, IL). $P<0.05$ was considered statistically significant.

\section{Results}

PGN up-regulates CHIP mRNA expression in macrophages

To determine whether CHIP mRNA expression is regulated by TLR stimulation, we treated RAW 264.7 cells with LPS (TLR4 agonist, 200 ng/ml), PGN (TLR2 agonist, 10 g/ml) 
Fig. 1. PGN upregulates CHIP mRNA expression in macrophages. RAW264.7 cells were treated with LPS (200ng/ml), PGN $(10 \mu \mathrm{g} / \mathrm{ml})$ and CpG ODN $(1 \mu \mathrm{g} / \mathrm{ml})$ for $0,0.5,2,4,8$, 12 hour after starvation for 8 hour. Expression of CHIP mRNA was determined by Real-time PCR and GAP$\mathrm{DH}$ expression served as the control. Data expressed as mean \pm SEM from three independent experiments. $* P<0.05$ versus 0 hour.
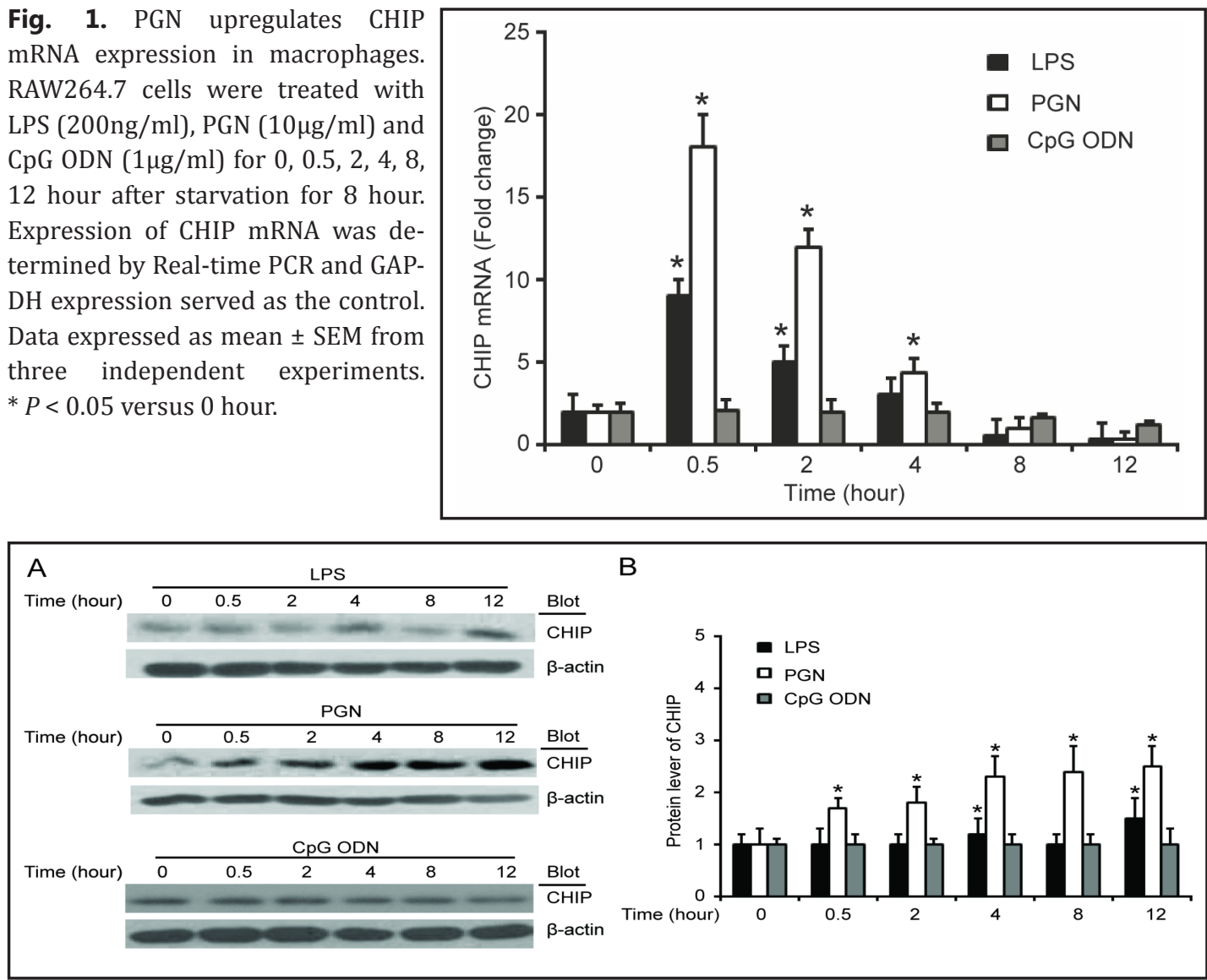

Fig. 2. PGN increases CHIP expression after stimulation in protein level. RAW264.7 cells were treated with LPS (200ng/ml), PGN $(10 \mu \mathrm{g} / \mathrm{ml})$, and CpG ODN $(1 \mu \mathrm{g} / \mathrm{ml})$ for 0, 0.5, 2, 4, 8, 12 hours after starvation for 8 hour. Proteins were extracted and analyzed CHIP expression using immunoblotting and $\beta$-actin expression served as the control (lower panel). Data expressed as mean \pm SEM from three independent experiments. $* P<0.05$ versus 0 hour.

and CpG ODN (TLR9 agonist, $1 \mu \mathrm{g} / \mathrm{ml}$ ) after overnight starvation. qPCR analysis revealed that both LPS and PGN significantly increased the expression of CHIP mRNA during 0.5-4 $\mathrm{h}$, and the peaks were at $2 \mathrm{~h}$. Moreover, the expression of CHIP was approximately 2 -fold higher in PGN-treated group than that in LPS group. In contrast, no significant change in CHIP mRNA was observed after CpG ODN treatment (Fig. 1). These results indicate that PGN may play an important role in regulating CHIP expression and activity.

\section{PGN increases CHIP expression in protein level after stimulation}

To further investigate whether TLR agonists increases CHIP expression at protein level, RAW 264.7 were treated with LPS (200 ng/ml), PGN $(10 \mu \mathrm{g} / \mathrm{ml})$ and CpG ODN $(1 \mu \mathrm{g} / \mathrm{ml})$ 0-12 h. Western blot analysis indicated that PGN treatment increased the levels of CHIP protein in a time-dependent manner. LPS treatment markedly enhanced CHIP protein levels at $12 \mathrm{~h}$. However, CpG ODN had no effect on CHIP protein expression (Fig. 2). These results suggest that PGN markedly up-regulates the protein expression of CHIP.

\section{PGN up-regulates CHIP expression through TLR2}

PGN is known to activate cells through TLR2, which results in the activation of downstream signal pathway [23]. To determine whether TLR2 is involved in the regulation of CHIP caused by PGN, peritoneal macrophage was isolated from WT and TLR2 KO mice and then stimulated by PGN for 0-8 h. PGN treatment markedly up-regulated the levels of 


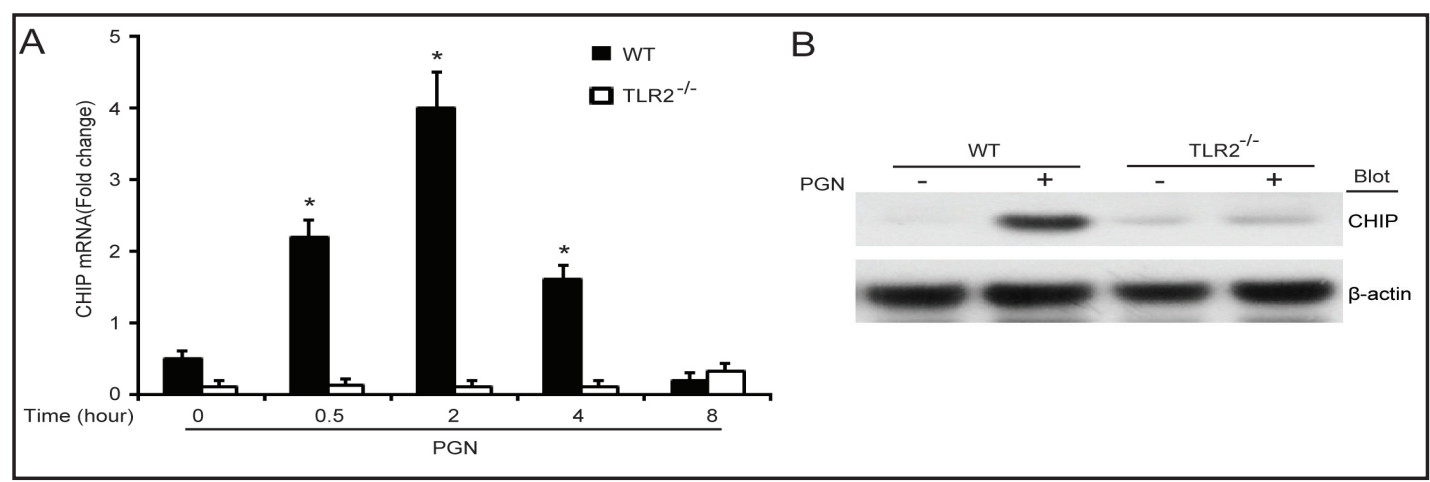

Fig. 3. PGN upregulates CHIP expression through TLR2. Both wild-type and TLR2 $\%$ peritoneal macrophages were treatment with PGN $(10 \mu \mathrm{g} / \mathrm{ml})$ for 0, 0.5, 2, 4, 8 hours after starvation. Expression of CHIP was determined by Real-time PCR (A) and Immunoblot(B). Data expressed as mean \pm SEM from threeindependentexperiments. $* P<0.05$ versus 0 hour.

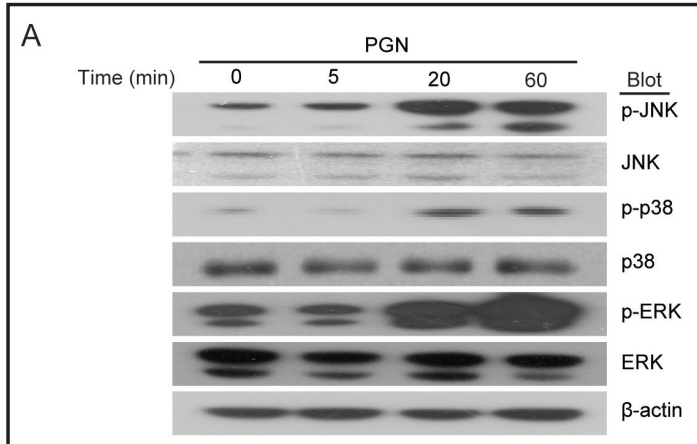

B

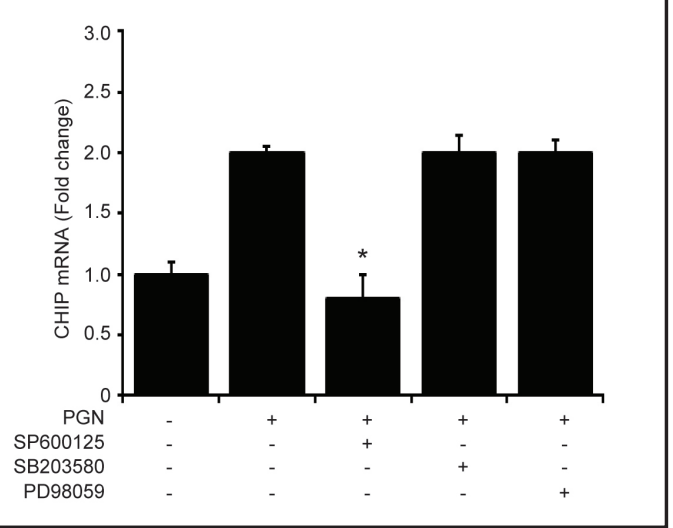

Fig. 4. PGN induces the expression of CHIP via JNK pathway. (A) PGN induced activation of the JNK, p38, ERK pathway. RAW264.7 cells were treated with $10 \mu \mathrm{g} / \mathrm{ml}$ of PGN for various periods of time. Phosphorylation of JNK, p38 and ERK were detected with Western bolt. (B) SP600125 inhibited PGN-induced CHIP upregulation in macrophage. Macrophages were pretreated with SP600125 (10 $\mathrm{mol} / \mathrm{L})$, SB203580 $(10 \mu \mathrm{mol} / \mathrm{L})$, PD98059 $(10 \mu \mathrm{mol} / \mathrm{L})$ for $30 \mathrm{~min}$ before 2 hours incubation with $10 \mu \mathrm{g} / \mathrm{ml}$ of PGN. Data expressed as mean \pm SEM from three independent experiments. ${ }^{*} P<0.05$ versus control.

CHIP mRNA compared with control during 0.5-4 h. In contrast, no change was observed in TLR2 KO macrophages (Fig. 3A). Moreover, the expression of CHIP protein was significantly increased in PGN-treated WT macrophages compared with untreated WT macrophage, whereas this effect was abolished in TLR2 KO macrophages (Fig. 3B). Thus, PGN-induced CHIP expression is TLR2-dependant.

\section{PGN induces the expression of CHIP via JNK pathway}

It is known that MAPK signaling pathways play critical roles in regulating the cell growth, proliferation and survival in various cell types [24]. Thus, we then investigated whether MAPKs (JNK, p38 and ERK) are involved in PGN-induced CHIP expression. We first examined the levels of JNK, p38 and ERK phosphorylation in RAW 264.7 after PGN $(10 \mu \mathrm{g} /$ $\mathrm{ml}$ ). Consistent with previous reports [25], we found that PGN treatment markedly increased the levels of JNK, p38 and ERK phosphorylation, which peaked at 20-60 min, indicating that PGN can induce activation of JNK, p38 and ERK signaling pathways in macrophages (Fig. 4A).

To further explore which signaling pathway contributes to PGN-induced CHIP expression, specific MAPK inhibitors SP600125 (JNK inhibitor, $10 \mu \mathrm{M}$ ), SB203580 (p38 


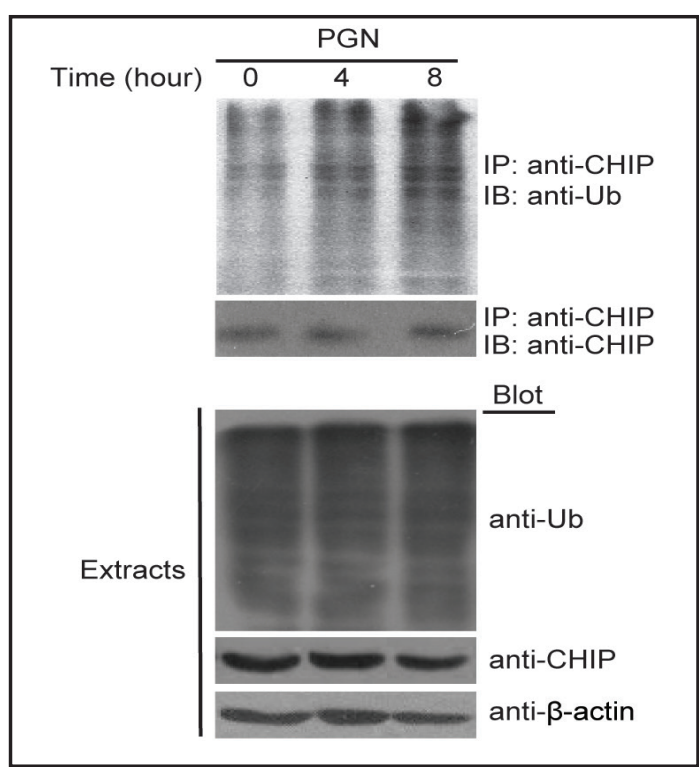

Fig. 5. PGN enhances ubiquitin ligase activity of CHIP. RAW264.7 treated with PGN $(10 \mu \mathrm{g} / \mathrm{ml})$ at the indicated points. Cell extracts were immunoprecipitated (IP) with anti-CHIP antibody and immunoblotted (IB) with an anti-Ub or anti-CHIP. The expression of CHIP in the cell extracts was determined with anti-CHIP antibody or $\beta$-actin.

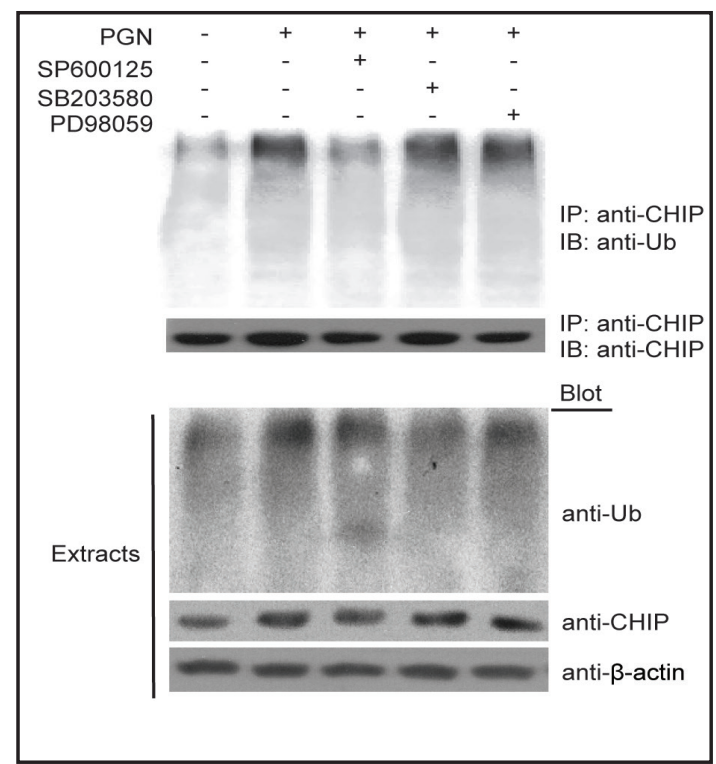

Fig. 6. PGN increases ubiquitin ligase activity of CHIP though JNK pathway. RAW264.7 cells were pretreated with SP600125 (10 $\mu \mathrm{mol} / \mathrm{L})$, SB203580 (10 $\mu \mathrm{mol} / \mathrm{L})$, PD98059 $(10 \mu \mathrm{mol} / \mathrm{L})$ for $30 \mathrm{~min}$ before incubation with $10 \mu \mathrm{g} / \mathrm{ml}$ of PGN for 8 hours. Cell extracts were immunoprecipitated (IP) with anti-CHIP antibody and immunoblotted (IB) with an anti-Ub or anti-CHIP. The expression of CHIP in the cell extracts was determined with anti-CHIP antibody or $\beta$-actin.

MAPK inhibitor, $10 \mu \mathrm{M}$ ) and PD98059 (ERK MAPK inhibitor, $10 \mu \mathrm{M}$ ) were used to treat cells for $30 \mathrm{~min}$ before 2 hour incubation with $10 \mu \mathrm{g} / \mathrm{ml}$ of PGN. As shown in Fig. 4B, SP600125 significantly inhibited PGN-induced expression of CHIP mRNA, whereas SB203580, PD98059 had no effect. Collectively, these data indicate that JNK pathway plays a critical role in PGNinduced expression of CHIP in macrophages.

\section{PGN enhances ubiquitin ligase activity of CHIP}

As an ubiquitin ligase, CHIP interaction with the molecular chaperones results in client substrate ubiquitination and degradation by the $26 \mathrm{~S}$ proteasome [26]. We then determined whether PGN affects the ubiquitin ligase activity of CHIP. After treatment with PGN at the indicated points, cell extracts were immunoprecipitated with anti-CHIP antibody and immunoblotted with an anti-Ub. We found that ubiquitination of CHIP increased in a timedependent manner following PGN treatment (Fig. 5). Thus, these data indicate that PGN can increase the ubiquitin ligase activity of CHIP in macrophages.

\section{PGN increases ubiquitin ligase activity of CHIP though JNK pathway}

We then investigated the molecular mechanism by which PGN increases ubiquitin ligase activity of CHIP in macrophages. Since PGN induces phosphorylation of JNK, p38, and ERK, we then used specific MAPK inhibitors to pretreat RAW 264.7 with SP600125 $(10 \mu \mathrm{M})$, SB203580 $(10 \mu \mathrm{M})$, PD98059 $(10 \mu \mathrm{M})$ for 30 min before 8 hours incubation with $10 \mu \mathrm{g} / \mathrm{ml}$ of PGN. As shown in Fig. 6, SP600125 significantly inhibited the PGN-induced ubiquitination of CHIP, whereas SB203580 and PD98059 had no effect after PGN stimulation. Together, these data indicate that JNK pathway plays a critical role in the PGN-induced increase in CHIP activity. 


\section{Discussion}

In this study, we for the first time demonstrated that the TLR2 agonist PGN stimulation drastically increased CHIP mRNA and protein expression in macrophages. These effects were predominantly mediated via the TLR2-JNK pathway. Moreover, PGN-induced activation of ubiquitin ligase activity of CHIP was markedly attenuated by JNK inhibitor. Thus, these results demonstrated that PGN regulates the expression and ubiquitin ligase activity of CHIP through JNK pathway.

CHIP is a U-box-dependent E3 ubiquitin ligase and cochaperone protein [12]. It contains three major structural domains. In addition to its well-characterized three-tetratricopeptide repeats (TRP) domain that allows it to interact with chaperones such as Hsc70/Hsp70 and Hsp90, it also has charged domain, and U-box domain that confers E3 ubiquitin ligase activity and facilitates the polyubiquitination of chaperone substrates [13]. Studies have shown that CHIP plays important roles in the regulation of pathophysiological processes through promoting the degradation of various target proteins including cystic fibrosis transmembrane conductance regulator (CFTR), ErBB2, Foxo1, myocardin, Ask1, p53, Tau, and ataxin 1 proteins $[27,28]$. Several studies have demonstrated that the expression of CHIP at mRNA and protein levels is regulated by various factors. Hypoxia, I/R injury, BAG2 and cochaperone HspBP1 inhibit the expression of ubiquitin ligase activity of CHIP. In contrast, heat shock and Ang II treatment have opposite effect [29, 30], but the underlying mechanisms remain poorly understood. Recently, it has been shown that CHIP plays an important role in regulating inflammatory responses. Overexpression of CHIP significantly suppressed Ang II-induced macrophage infiltration and expression of proinflammatory cytokines though NF- $\kappa B$ and MAPK signaling pathways [17]. CHIP also participates in innate immunity through TLR4 and TLR9 [16]. However, whether TLR signaling pathway regulates CHIP expression and ligase activity of CHIP and the underlying mechanisms remain to be explored.

TLRs family includes several receptors, including TLR2, which plays important roles in cardiovascular diseases such as infarction, restenosis, atherosclerosis and cardiomyopathy [31]. PGN is a major cell wall component of Gram-positive bacteria. Unlike LPS that is recognized by TLR4, PGN is recognized by TLR2 and then activates MAPKs pathway and induces production of inflammatory cytokines [31]. The MAPKs, including JNK, p38, and ERK, play an important role in regulating macrophage to induce the production of inflammatory cytokines during various pathological insults. It has been shown that PGN induces MAPKs activation in macrophage, thereby leads to expression of inducible nitric oxide synthase and NO [9]. However little is understood about the role of PGN in the regulation of CHIP. In this study, our data indicated that TLR2 agonist PGN dramatically increased CHIP expression in mRNA and protein levels (Fig. 1 and 2), whereas this effect of PGN on CHIP expression was abolished in TLR2 KO macrophages (Fig. 3). Moreover, specific inhibition of JNK with SP600125 markedly attenuated PGN-induced expression of CHIP, whereas a specific p38 MAPK and ERK inhibitors had no effect (Fig. 4B).These data suggest that activation of JNK is required for PGN-induced expression of CHIP in macrophages.

Increasing evidence suggests that the ubiquitin-proteasome system is responsible for the degradation of misfolded proteins [32]. The process of protein degradation by ubiquitination is mediated by enzymes known as E1, E2, and E3. As an E3 ligase, we wondered whether the increased expression of CHIP induced by PGN could associate with the increased ubiqitin ligase activity of CHIP. We found that PGN treatment also enhanced CHIP ligase activity in a time-dependent manner (Fig. 5) and this action was attenuated by SP600125, an inhibitor of JNK pathway. These data further demonstrate that PGN up-regulates both expression of CHIP and ubiqitin ligase activity through JNK signaling pathway in macrophages.

In conclusion, we provide novel evidence that TLR2 agonist PGN promoted the expression and ubiquitin ligase activity of CHIP in macrophages via JNK signaling pathway. Thus, the present study has identified a novel mechanism of regulation of CHIP expression and activity by PGN, which implies a novel therapeutic role of JNK inhibition during bacterial 


\begin{tabular}{|c|c|c|}
\hline Cellular PI & Cell Physiol Biochem 2013;32:1097-1105 & \\
\hline and Biochemistry & $\begin{array}{l}\text { Dol: 10.1159/000354509 } \\
\text { Publisnea onine. vovember 08, } 2013\end{array}$ & $\begin{array}{l}\text { O } 2013 \text { S. Karger AG, Basel } \\
\text { www.karger.com/cpb }\end{array}$ \\
\hline
\end{tabular}

infection. However, future study is required to identify the immediate target of JNK kinase activity which promotes the expression and activity of CHIP, and its role in regulating TRL2mediated actions in cardiovascular diseases.

\section{Acknowledgements}

This work was supported by grants from the National Natural Science Foundation of China (81025001, 81330003 and 81100183), Chang Jiang Scholar Program and the Beijing High-level Talents Program (HL, PH20110507).

\section{References}

1 Martin GS, Mannino DM, Eaton SMoss M: The epidemiology of sepsis in the United States from 1979 through 2000. N Engl J Med 2003;348:1546-1554.

2 Kawai TAkira S: The role of pattern-recognition receptors in innate immunity: update on Toll-like receptors. Nat Immunol 2010;11:373-384.

-3 Shockman GD, Daneo-Moore L, Kariyama RMassidda O: Bacterial walls, peptidoglycan hydrolases, autolysins, and autolysis. Microb Drug Resist 1996;2:95-98.

4 Michelsen KS, Aicher A, Mohaupt M, Hartung T, Dimmeler S, Kirschning CJSchumann RR: The role of tolllike receptors (TLRs) in bacteria-induced maturation of murine dendritic cells (DCS). Peptidoglycan and lipoteichoic acid are inducers of DC maturation and require TLR2. J Biol Chem 2001;276:25680-25686.

-5 Derijard B, Raingeaud J, Barrett T, Wu IH, Han J, Ulevitch RJDavis RJ: Independent human MAP-kinase signal transduction pathways defined by MEK and MKK isoforms. Science 1995;267:682-685.

6 Eda H, Shimada H, Beidler DRMonahan JB: Proinflammatory cytokines, IL-1beta and TNF-alpha, induce expression of interleukin-34 mRNA via JNK- and p44/42 MAPK-NF-kappaB pathway but not p38 pathway in osteoblasts. Rheumatol Int 2011;31:1525-1530.

7 Hsieh IN, Chang AS, Teng CM, Chen CCYang CR: Aciculatin inhibits lipopolysaccharide-mediated inducible nitric oxide synthase and cyclooxygenase-2 expression via suppressing NF-kappaB and JNK/p38 MAPK activation pathways. J Biomed Sci 2011;18:28.

8 Chen CC, Tsai PC, Wei BLChiou WF: 8-Prenylkaempferol suppresses inducible nitric oxide synthase expression through interfering with JNK-mediated AP-1 pathway in murine macrophages. Eur J Pharmacol 2008;590:430-436.

-9 Bhatt KH, Sodhi AChakraborty R: Role of mitogen-activated protein kinases in peptidoglycan-induced expression of inducible nitric oxide synthase and nitric oxide in mouse peritoneal macrophages: extracellular signal-related kinase, a negative regulator. Clin Vaccine Immunol 2011;18:994-1001.

10 Wang C, Chen T, Zhang J, Yang M, Li N, Xu XCao X: The E3 ubiquitin ligase Nrdp1 'preferentially' promotes TLR-mediated production of type I interferon. Nat Immunol 2009;10:744-752.

11 Liu GY, Liu SX, Li P, Tang L, Han YM, An HZ, Li JY, Dai XK, Li N, Cao XTYu YZ: Cloning and characterization of DULP, a novel ubiquitin-like molecule from human dendritic cells. Cell Mol Immunol 2009;6:27-33.

12 Ballinger CA, Connell P, Wu Y, Hu Z, Thompson LJ, Yin LYPatterson C: Identification of CHIP, a novel tetratricopeptide repeat-containing protein that interacts with heat shock proteins and negatively regulates chaperone functions. Mol Cell Biol 1999;19:4535-4545.

13 Dai Q Zhang C, Wu Y, McDonough H, Whaley RA, Godfrey V, Li HH, Madamanchi N, Xu W, Neckers L, Cyr DPatterson C: CHIP activates HSF1 and confers protection against apoptosis and cellular stress. EMBO J 2003;22:5446-5458.

14 Xie P, Fan Y, Zhang H, Zhang Y, She M, Gu D, Patterson CLi H: CHIP represses myocardin-induced smooth muscle cell differentiation via ubiquitin-mediated proteasomal degradation. Mol Cell Biol 2009;29:23982408. 


\section{Cellular Physiology $\quad$ Cell Physiol Biochem 2013;32:1097-1105 and Biochemistry

15 Jang KW, Lee KH, Kim SH, Jin T, Choi EY, Jeon HJ, Kim E, Han YSChung JH: Ubiquitin ligase CHIP induces TRAF2 proteasomal degradation and NF-kappaB inactivation to regulate breast cancer cell invasion. J Cell Biochem 2011;112:3612-3620.

16 Yang M, Wang C, Zhu X, Tang S, Shi L, Cao XChen T: E3 ubiquitin ligase CHIP facilitates Toll-like receptor signaling by recruiting and polyubiquitinating Src and atypical PKC\{zeta\}. J Exp Med 2011;208:2099-2112.

17 Yang K, Zhang TP, Tian C, Jia LX, Du JLi HH: Carboxyl terminus of heat shock protein 70-interacting protein inhibits angiotensin II-induced cardiac remodeling. Am J Hypertens 2012;25:994-1001.

$\checkmark 18$ Xu CW, Zhang TP, Wang HX, Yang HLi HH: CHIP enhances angiogenesis and restores cardiac function after infarction in transgenic mice. Cell Physiol Biochem 2013;31:199-208.

19 Li H, Kim WJ, Jiang J, Lee SH, Youn HS, Moon EY, Kim TJ, Ye SK, Ryu JH, Kang TB, Koppula S, Park PJ, Choi DKLee KH: MyD88-dependent Toll-like receptor signaling is required for murine macrophages response to IS2. Int Immunopharmacol 2011;11:1578-1583.

20 Li Y, Zhang C, Wu Y, Han Y, Cui W, Jia L, Cai L, Cheng J, Li HDu J: Interleukin-12p35 deletion promotes CD4 T-cell-dependent macrophage differentiation and enhances angiotensin II-Induced cardiac fibrosis. Arterioscler Thromb Vasc Biol 2012;32:1662-1674.

21 Li HH, Willis MS, Lockyer P, Miller N, McDonough H, Glass DJPatterson C: Atrogin-1 inhibits Akt-dependent cardiac hypertrophy in mice via ubiquitin-dependent coactivation of Forkhead proteins. J Clin Invest 2007;117:3211-3223.

22 Murata S, Chiba TTanaka K: CHIP: a quality-control E3 ligase collaborating with molecular chaperones. Int J Biochem Cell Biol 2003;35:572-578.

23 Schwandner R, Dziarski R, Wesche H, Rothe MKirschning CJ: Peptidoglycan- and lipoteichoic acid-induced cell activation is mediated by toll-like receptor 2. J Biol Chem 1999;274:17406-17409.

24 Geest CRCoffer PJ: MAPK signaling pathways in the regulation of hematopoiesis. J Leukoc Biol 2009;86:237-250.

25 Shepherd EG, Zhao Q, Welty SE, Hansen TN, Smith CVLiu Y: The function of mitogen-activated protein kinase phosphatase-1 in peptidoglycan-stimulated macrophages. J Biol Chem 2004;279:54023-54031.

26 McDonough HPatterson C: CHIP: a link between the chaperone and proteasome systems. Cell Stress Chaperones 2003;8:303-308.

-27 Shaked H, Shiff I, Kott-Gutkowski M, Siegfried Z, Haupt YSimon I: Chromatin immunoprecipitation-on-chip reveals stress-dependent p53 occupancy in primary normal cells but not in established cell lines. Cancer Res 2008;68:9671-9677.

28 Li F, Xie P, Fan Y, Zhang H, Zheng L, Gu D, Patterson CLi H: C terminus of Hsc70-interacting protein promotes smooth muscle cell proliferation and survival through ubiquitin-mediated degradation of Fox01. J Biol Chem 2009;284:20090-20098.

29 Alberti S, Bohse K, Arndt V, Schmitz AHohfeld J: The cochaperone HspBP1 inhibits the CHIP ubiquitin ligase and stimulates the maturation of the cystic fibrosis transmembrane conductance regulator. Mol Biol Cell 2004;15:4003-4010.

-30 Arndt V, Daniel C, Nastainczyk W, Alberti SHohfeld J: BAG-2 acts as an inhibitor of the chaperone-associated ubiquitin ligase CHIP. Mol Biol Cell 2005;16:5891-5900.

-31 Frantz S, Ertl GBauersachs J: Mechanisms of disease: Toll-like receptors in cardiovascular disease. Nat Clin Pract Cardiovasc Med 2007;4:444-454.

32 Willis MSPatterson C: Into the heart: the emerging role of the ubiquitin-proteasome system. J Mol Cell Cardiol 2006;41:567-579. 